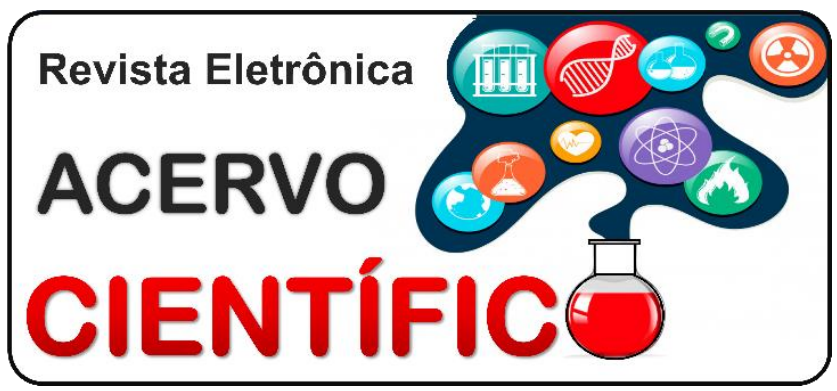

\section{REVISÃO BIBLIOGRÁFICA}

Recebido em: 8/2020

Aceito em: 7/2020

Publicado em: 12/2020

\title{
Cirurgia bariátrica por bypass gástrico em $Y$ de Roux: abordagem da técnica e de possíveis complicações tardias no pós-operatório
}

\author{
Bariatric surgery by gastric bypass in $Y$ of Roux: approach of the technique and possible \\ late complications in the post-operatory
}

\section{Cirugía bariátrica por bypass gástrico en Y de Roux: enfoque de la técnica y posibles complicaciones tardías en el post-operatorio}

Rayane Cristina Batista Rodrigues ${ }^{1 *}$, Denes Marques de Figueiredo ${ }^{2}$, Amanda Carvalho Mesquita ${ }^{3}$, Esley Ruas Alkimim", Fabio Henrique da Silveira Peixoto' ${ }^{1}$, João Victor de Paula Leite ${ }^{5}$, Julia Azevedo Penha ${ }^{3}$, Larissa Rezende Lima Pereira ${ }^{1}$, Melina Bequer de Sousa ${ }^{6}$, Niccoly Kolle Pereira ${ }^{7}$.

\begin{abstract}
Resumo: Esse artigo buscou apresentar possíveis complicações pós-operatórias que se desenvolvem tardiamente após a realização da cirurgia bariátrica pela técnica bypass gástrico com anastomose em $Y$ de Roux (BGYR) e os fatores que viabilizam minimizar essas intercorrências para que resulte em um prognóstico favorável. O estudo evidencia a importância dessa intervenção invasiva para o tratamento da obesidade e do sobrepeso, uma vez que são problemas crescentes e estão fortemente ligados a comorbidades que influenciam na morbimortalidade da população acometida. Discutem-se aspectos relevantes dos procedimentos cirúrgicos bariátricos gerais, quanto às classificações, características e recomendações avaliativas - indicações e contraindicações - para a admissão da cirurgia. O enfoque do trabalho voltou-se à técnica BGYR, cujo procedimento cirúrgico é apresentado de forma mais detalhada, além da demonstração das principais manifestações clínicas, diagnóstico e como são desenvolvidas as complicações pósoperatórias tardias recorrentes. Também, são vistos fatores relevantes que auxiliam no reconhecimento dessas complicações, assim como intervenções e formas de prevenção importantes para um bom resultado da técnica. Por fim, considera-se a importância do procedimento e das questões levantadas quanto às complicações e o manejo correto, objetivando contribuir positivamente para a abordagem.
\end{abstract}

Palavras-chave: Bypass gástrico, Cirurgia bariátrica, Complicações pós-operatórias.

\footnotetext{
Abstract: This article aimed to present possible postoperative complications that develop late after bariatric surgery by gastric by pass technique with Roux-en-Y anastomosis (BGYR) and the factorsthat make it possibleto minimize these complications to results in a favorable prognosis. The study high lights the importance of this invasive intervention for the treatment of obesity and over weight, since they are increasing

${ }^{1}$ Centro Universitário de Caratinga (UNEC), Caratinga - MG. *E-mail: rayanecbr1507@gmail.com.

2Universidade Federal de Juiz de Fora (UFJF), Juiz de Fora - MG.

${ }^{3}$ Centro Universitário de Belo Horizonte (UniBH), Belo Horizonte - MG.

${ }^{4}$ Faculdades Unidas do Norte de Minas (FUNORTE), Montes Claros - MG.

5 Universidade Federal do Triângulo Mineiro (UFTM), Uberaba - MG.

6Universidade de Rio Verde (Unirv), Rio Verde - GO.

${ }^{7}$ Universidade Cidade de São Paulo (UNICID), São Paulo - SP.
} 
problems and are strongly linked to comorbidities that influence the morbidity and mortality of the affected population. Relevant aspects of general bariatric surgical procedures are discusse dregarding evaluation classifications, characteristics and recommendations -indications and contraindications - for admission of surgery. The focus of the work was turned to the BGYR technique, who sesurgical procedure is presented in more detail, in addition to the demonstration of the main clinical manifestations, diagnosis and how recurrent late postoperative complications are developed. Relevant factors are seen that assist in there cognition of these complications, as well as interventions and forms of prevention that are important for a good result of the technique. Finally, we consider the importance of the procedure and the questions raise dregarding complications and the correct management, aiming to contribute positively to the approach.

Keywords: Gastric bypass, Bariatric surgery, Postoperative complications.

Resumen: Este artículo pretendía presentar las posibles complicaciones post-operatorias que se desarrollan trás la cirugía bariátrica por la técnica de bypass gástrico com anastomosis en Y de Roux (BGYR), los factores que permiten minimizar complicaciones para que el pronóstico sea favorable. El estudio se destaca la importancia de intervención invasiva para el tratamiento de la obesidad y sobrepeso, que son problemas cada vez mayores y están fuertemente vinculados a las comorbilidades que influyen en la morbilidad y mortalidad de la población afectada. Se discuten los aspectos relevantes de los procedimientos cirúrgicos bariátricos generales, cuanto a clasificaciones, características y recomendaciones de evaluación, para la admisión de la cirugía. El estúdio centro em la técnica de la BGYR, cuyo procedimento quirúrgico se presenta con más detalle, además de la demostración de las principales manifestaciones clínicas, el diagnóstico y la forma en que se desarrollan las complicaciones post-operatorias tardias recurrentes. Además, observan factores pertinentes que ayudan a reconocer essas complicaciones, así como intervenciones y formas de prevención importantes para um buen resultado de la técnica. Por último, se considera la importância del procedimiento y las cuestiones planteadas em relación com las complicaciones y el manejo correcto, com el fin de contribuir positivamente al enfoque.

Palabras-chave: Bypass gástrico, Cirugía bariátrica, Complicaciones postoperatorias.

\section{INTRODUÇÃO}

Nas últimas décadas, a obesidade e o sobrepeso são problemas crescentes em todo o mundo, o que representa um desafio para a saúde pública. Essas condições afetam cerca de 36,9\% dos homens e 38\% das mulheres no mundo ocidental. A morbimortalidade dessa problemática relaciona-se ao grande número de comorbidades associadas, como a diabetes mellitus tipo 2, hipertensão arterial sistêmica, dislipidemia, câncer e outros. Logo, faz com que a busca por tratamentos cirúrgicos seja cada vez maior (MARTIGELONCH L, et al., 2019).

Por ser uma doença crônica e multifatorial seu principal tratamento é clínico e acompanhado por uma equipe multidisciplinar. No entanto, muito dos pacientes possuem dificuldades em aderir aos tratamentos não invasivos, como reeducação alimentar, medicamentos e exercícios físicos, de forma efetiva. Sendo assim, torna-se necessário uma intervenção invasiva, como a realização de procedimentos cirúrgicos.

Desse modo, ao considerar a forma cirúrgica como tratamento aos que não conseguem perder peso corporal e manter essa perda - mais de $5 \%$ do peso corporal mantido por mais de cinco anos - iniciou-se 0 desenvolvimento de técnicas de cirurgias bariátricas que favorecem essa perda com o intuito de evitar intercorrências e métodos ineficazes à diminuição de peso corporal (ZEVE JLM, et al., 2012).

As cirurgias bariátricas se dividem em restritivas, disabsortivas e mistas. Estas apresentam diferentes tipos de técnicas que são realizadas de acordo com a necessidade dos pacientes. As restritivas utilizam técnicas que alteram o tamanho e a capacidade do estômago, limitando o volume de alimento sólido que o paciente ingere, e, consequentemente, induzem a sensação de saciedade de forma precoce. As cirurgias disabsortiva são técnicas realizadas que alteram pouco o estômago, mas alteram essencialmente a absorção a nível do intestino delgado. Assim, vê se a prevalência do intuito de diminuir a absorção no organismo por alterar o 
tempo do trânsito intestinal, mediante o grande desvio do intestino. Já as cirurgias mistas associam restrição e disabsorção, uma vez que causam restrição na capacidade de receber o alimento pelo estômago que se encontra reduzido e possui um desvio curto do intestino com discreta má absorção de alimentos (MANCINI MC, 2006; ZEVE JLM, et al., 2012). Nesse sentido, a cirurgia bariátrica apresenta-se como terapêutica eficiente, tanto para a perda do excesso de peso, como para a resolutividade das comorbidades associadas. Entre os procedimentos cirúrgicos, o bypass gástrico em $Y$ de Roux (BGYR) é o método mais realizado em todo o mundo, pertencendo ao grupo de cirurgias combinadas/mistas, uma vez que gera restrição e máabsorção (BARROS F, et al., 2019).

Apesar da técnica cirúrgica BGYR possuir uma segurança bem documentada, várias complicações podem ocorrer em diferentes graus de morbidade e mortalidade. Essas complicações podem ser divididas em precoces; e tardias quando ocorrem após 30 dias da cirurgia, por exemplo a estenose de anastomose, a ulceração marginal e a fístula gastro gástrica. Tais complicações possuem causalidades multifatoriais e dependem em sua maioria, de fatores intrínsecos de cada paciente, assim como da técnica empregada (US DE PAZ, et al., 2015; ACQUAFRESCA PA, et al., 2015; PALERMO M, et al., 2015).

Percebe-se que devido à sua maior utilização como escolha terapêutica, e a dificuldade de manejo clínico decorrente das características clínicas que os pacientes submetidos podem apresentar, as complicações advindas das operações bariátricas são crescentes. Assim, diante o contexto, o presente estudo possui como objetivo o levantamento de possíveis complicações após a realização do procedimento bypass gástrico em $Y$ de Roux, cujo enfoque principal do trabalho volta-se às complicações tardias. Também, o trabalho possui como orientação questionamentos sobre o correto desenvolvimento da técnica e a sua contribuição para um favorável prognóstico pós-operatório (WRZESINSKI A, et al., 2015).

\section{REVISÃO BIBLIOGRÁFICA}

\section{Generalidades, indicações e contraindicações da cirurgia bariátrica}

A obesidade é considerada uma doença crônica que cresce mundialmente, e relaciona-se, em especial, a fatores predisponentes como hábitos alimentares, sedentarismo e tabagismo. Mediante a publicação da Portaria no 424 de 19 de Março de 2013, o Ministério da Saúde redefiniu as diretrizes no que tange à organização da prevenção e do tratamento da obesidade como um caminho prioritário da Rede de Atenção à Saúde das Pessoas com Doenças Crônicas, de modo a reforçar a importância da Atenção Primária de Saúde (APS) no cuidado integral de pacientes portadores dessa doença (MS, 2013). Concomitantemente, as cirurgias bariátricas são consideradas como principais procedimentos cirúrgicos realizados para o tratamento da perda de peso corporal e redução de comorbidades de pessoas acometidas pela obesidade (JARA R, 2017; MARAMBIO A, 2015).

Segundo a Sociedade Brasileira de Cirurgia Bariátrica e Metabólica (SBCBM), existem alguns métodos de avaliação responsáveis pela admissão de uma cirurgia bariátrica através de indicações e contraindicações pré-estabelecidas. Os pré-requisitos para as indicações da cirurgia envolvem: IMC $\geq 40 \mathrm{~kg} / \mathrm{m}^{2}, I \mathrm{IMC} \geq 35 \mathrm{~kg} / \mathrm{m}^{2} \mathrm{e}$ comorbidades associadas, tratamento clínico prévio não eficaz por pelo menos 2 anos, avaliação do estado mental saudável, compreensão pelo paciente e pela família acerca dos riscos e idade entre 16 a 65 anos. Em contrapartida, as contraindicações pontuam: pacientes com obesidade advinda de alguma doença do sistema endócrino, jovens em fase de crescimento longitudinal, indivíduos com distúrbios mentais, psicóticos ou demenciados graves, indivíduos com história recente de tentativa de suicídio e dependentes químicos. Essas recomendações devem ser seguidas corretamente, com o objetivo de priorizar a segurança do paciente diante do procedimento e reduzir as taxas de complicações e mortalidade (SBC BM, 2017).

\section{A técnica bypass gástrico em $Y$ de Roux}

O bypass gástrico em $Y$ de Roux (BGYR), classificado como misto, é considerado procedimento padrãoouro dentre as intervenções cirúrgicas bariátricas e destaca-se como uma das principais técnicas utilizadas para o tratamento da obesidade (WRZESINSKI A, et al., 2015). Essa técnica pode ser executada através da laparoscopia, com o método primordial da lise de aderências entre o estômago e o fígado. A seguir, é realizado o procedimento da retração do fígado, da dissecção do omento menor e da mobilização do tubo gástrico em 
sentido ao diafragma. Adiante, o tubo gástrico é fragmentado de maneira horizontal por um grampeador. Assim, outro grampeamento $(60 \mathrm{~cm})$ é realizado de forma vertical, com o objetivo de reduzir a nova bolsa lateralmente. Nesse contexto, a alça alimentar $Y$ de Roux é elaborada através de uma única anastomose gastrojejunal (ligação de uma porção do estômago ao jejuno). Posteriormente, ela é medida e uma jejunojejunostomia mecânica laterolateral é realizada cerca de 150 e $250 \mathrm{~cm}$ do Ligamento de Treitz (ZORRON R, et al., 2016; GUIRAT A, 2018).

Por pertencer ao grupo dos procedimentos combinados, pode cursar com restrição e disabsorção. Essa restrição é provocada pela exérese do estômago proximal, limitando desse modo o seu volume. Logo, é necessário deixar a porção remanescente do estômago excluída, através da criação de uma bolsa de cerca de 10 a $25 \mathrm{ml}$. Em contrapartida, a má-absorção é gerada por meio da divisão do intestino delgado formando uma alça alimentar (alça de Roux) e outra biliopancreática (ACQUAFRESCA PA, et al., 2015).

\section{Complicações tardias recorrentes e os aspectos relevantes envolvidos}

É imprescindível compreender aspectos importantes relacionados aos tipos de complicações que podem incidir após a realização de cirurgias bariátricas, sobretudo da técnica por bypass gástrico em $Y$ de Roux. $O$ entendimento desses acometimentos elucida questões como a razão custo e benefício do procedimento e também auxilia no diagnóstico das complicações pelo profissional de saúde especializado (VIEIRA E, 2018).

Após a realização da técnica BGYR é recorrente a existência de complicações tardias, tornando-se dever do cirurgião estar preparado para resolvê-las. Esses acometimentos manifestam-se com diversos graus de morbidade e mortalidade. Dentro desse viés, destacam-se as seguintes possíveis complicações tardias recorrentes, as quais são alvo desse estudo: estenose de anastomose gastro jejunal, fístula gastro gástrica, hérnias internas, úlcera marginal e deficiências nutricionais (WRZESINSKI A, et al., 2015; US De PAZ G, 2015).

\section{Estenose de Anastomose Gastrojejunal}

Está relacionada a problemas técnicos que provocam tensão e isquemia na região anastomótica vinculado ao incorreto uso de métodos de calibração e de sutura no decorrer do processo. Essa complicação apresenta grande incidência na abordagem laparoscópica em relação à abordagem aberta.

O diagnóstico é feito através da clínica - náuseas e vômitos pós-prandiais, refluxo gastroesofágico e disfagia parcial ou total -, associada a exames complementares, como endoscopia digestiva alta ou radiografia contrastada. A endoscopia consiste no método de escolha, devido à sua maior sensibilidade, além da possibilidade de tratamento da estenose; realizado com a dilatação endoscópica com uso de balão pneumático, com resolução de 95\% dos casos (SAMPAIO-NETO J, et al., 2016; US De PAZ G, 2015).

Revisões sistemáticas apontam que, além do tempo cirúrgico, o índice de estenose de anastomose gastrojejunal é mais frequente com o uso de grampeadores circulares em comparação aos lineares. Estes não apresentaram relação com o aparecimento de fístulas, sangramento pós-operatório e úlceras marginais. Não existe protocolo bem estabelecido para resolubilidade dessa complicação. Primordialmente o objetivo é o alívio dos sintomas, porém, para ocorrer a perda de peso a anastomose deve-se manter estreita. Quando a dilatação endoscópica não apresenta resultado significativo, a operação de anastomose pode ser necessária, contudo, se caracteriza com alta complexidade (SAMPAIO-NETO J, et al., 2016; US De PAZ G, 2015).

\section{Fístula Gastro-Gástrica}

Fístula Gastro-Gástrica (FGG) é uma comunicação atípica entre a bolsa gástrica e o estômago excluído. Dentre as possíveis etiologias que visam explicar o desencadeamento dessa condição, destacam-se: a iatrogenia, a deiscência anastomótica, o tipo operatório realizado, a úlcera marginal ou perfurada, a erosão de corpo estranho e a tendência natural de migração da porção gástrica em se conectar à parte remanescente. Quanto aos sintomas, ressaltam-se o ganho ou perda de peso de forma inadequada, o desenvolvimento de ulceração, a presença de sangramento recorrente, dor abdominal e formação de estenoses (PALERMO M, et al., 2015; US De PAZ G, 2015). 
Tornam-se necessárias técnicas anastomóticas cuidadosas, suturas meticulosas e a escolha de materiais pertinentes e de qualidade excelente para a cirurgia. Além disso, mediante o quadro de FGG, a intervenção terapêutica é variável, sendo o tratamento com a utilização de fármacos inibidores de bomba de prótons e sucralfato uma opção com $37 \%$ de resolubilidade dos casos. No entanto, pacientes sintomáticos, mesmo após o uso de medicação adequada, carece da realização de cirurgia com intuito de fechamento ou exclusão do trato fistuloso, em que as principais abordagens descritas são: laparoscopia, ressecção do remanescente gástrico e alternativas que utilizam a endoscopia (PALERMO M, et al., 2015; US De PAZ G, 2015).

\section{Hérnias Internas}

Hérnia interna é uma complicação relativamente comum em pacientes que se submetem a cirurgias abdominais, e possui como fatores de risco a idade, ausência de hipertensão, cirurgia antirrefluxo anterior e grande perda de peso em um espaço curto de tempo (EDERVEEN JC, et al., 2018). As hérnias internas podem ser ocasionadas durante a criação das anastomoses no procedimento cirúrgico ou devido a defeitos mesentéricos e elas podem ser responsáveis por volvos e isquemia da alça de Roux. As manifestações clínicas costumam ocorrer no pós-operatório tardio, com quadro clínico inespecífico, com dor abdominal pós prandial, náuseas, vômitos e edema (EDERVEEN JC, et al., 2018).

O diagnóstico pode ser confirmado através de Tomografia Computadorizada do abdômen, que tem como achados comuns: distensão abdominal no andar superior do abdômen, localização do segmento herniado de alça jejunal acima do nível gástrico, rotação dos vasos mesentéricos acompanhada de densificação da gordura mesenterial, trajeto descendente do íleo médio/distal a partir do hipocôndrio esquerdo e o deslocamento anterior e para a direita do ângulo de Treitz (XIMENES MAS, et al., 2008).

\section{Úlcera marginal (UM)}

As úlceras marginais são úlceras pépticas que acometem a mucosa jejunal adjacente à anastomose gastrojejunal. A prevalência dessa complicação varia de $1 \%$ a $16 \%$ em pacientes submetidos ao Bypass Gástrico e o tempo médio transcorrido entre o bypass e a perfuração é em torno de 27 meses. Essa complicação tardia possui origem multifatorial e pode estar relacionada com o tamanho da bolsa gástrica e sua orientação, isquemia de mucosa e linha de grampeamento rompida. Além disso, estudos recentes mostraram a presença de Helicobacterpylorino pré-operatório e que essa bactéria é potencial causadora de ulcerações prévias que se complicam após a cirurgia. É válido ressaltar, também, que as ulcerações podem aparecer devido à irritação prolongada por material estranho no local da anastomose gastrojejunal, como os fios de sutura não absorvíveis. (PALERMO M, 2015; MARAMBIO A, 2015; ADOLFO GP, 2015; MALA T, 2018).

As úlceras marginais podem ser tratadas majoritariamente de maneira farmacológica utilizando os IBP's (Inibidores de Bomba de Prótons) e sucralfatos, bem como medidas não farmacológicas quando se previne os fatores desencadeantes dessa patologia. Ainda que, em suma, sejam tratadas clinicamente, existe uma grande parte dos pacientes que necessitam de intervenção cirúrgica devido às perfurações, úlceras crônicas e refratárias e também por lesões gastrointestinais associadas (PYKE O, et al., 2019).

\section{Deficiências nutricionais}

As deficiências nutricionais (DN) são normalmente encontradas em pacientes que foram submetidos à cirurgia bariátrica. Notoriamente, esse tipo de cirurgia promove alterações anatômicas e fisiológicas extensas no trato gastrointestinal. Dentre elas, pode haver diminuição da absorção da vitamina B12, como também carência de ferro e folato - que são absorvidos na porção duodenal do intestino delgado - cursando com anemia. Essas podem ser citadas como as principais deficiências encontradas após a bariátrica (SOUZA N, et al., 2020; BAILLY L, 2018).

Os procedimentos com desvio intestinal são potencializadores de deficiências nutricionais devido a uma dieta restritiva e hipocalórica. O BGYR aumenta, ainda mais, o comprometimento na absorção da Cobalamina (Vitamina B12) em virtude da insuficiência ou nenhum ácido presente na bolsa gástrica. Além disso, também há diminuição da produção do Fator Intrínseco (produzido pelas células parietais) que é responsável por auxiliar na absorção de B12 intestinal. Com isso, a suplementação de macronutrientes é recomendada pós 
cirurgias bariátricas para um melhor resultado cirúrgico e diminuição de futuras complicações (KORNERUP L, 2019; NIRUJOGI V, 2015).

\section{Fatores importantes para um bom prognóstico}

Segundo dados publicados pelo Colégio Americano de Cirurgiões $11,8 \%$ dos pacientes submetidos a cirurgia em $\mathrm{Y}$ de Roux apresentam complicações pós operatórias, que estão associadas à inúmeros custos, tanto pela necessidade de realizar novos procedimentos, como pela incapacitação temporária para exercer atividades laborais. Somado a isso há o prejuízo no bem estar do paciente, que muitas vezes já tem certo grau de debilitação (ABRAHAM CR, et al., 2015). Desse modo, torna-se pertinente a investigação das variáveis envolvidas no sucesso cirúrgico, para que se possa desenvolver estratégias preventivas para tais eventos adversos, já que se trata de um procedimento que promove melhora da qualidade de vida e de comorbidades que possuem relação com o excesso de peso (STOLL A, et al., 2016).

A utilização da técnica operatória correta pelos cirurgiões é de extrema importância. Os profissionais devem estar atentos aos detalhes pois o manejo cirúrgico adequado está relacionado ao menor índice de complicações cirúrgicas, além de melhor qualidade e expectativa de vida dos pacientes submetidos ao bypass gástrico (ACQUAFRESCA PA, et al., 2015). As complicações pós-operatórias podem ser evitadas utilizandose da técnica operatória correta e expertise do médico ao que diz respeito à escolha do tamanho e tipo dos grampos e no local do seu disparo, é necessário, também, reforçar a linha grampeada com material biológico, uso de selante de fibrina quando disponível, testar vazamentos no intra-operatório, evitar anastomose sob tensão e tomar os cuidados adequados para não ocasionar isquemia no Y de Roux laparoscópico (PALERMO M, 2015; ADOLFO G, 2015; MALA T, 2018).

Após o bypass gástrico em $\mathrm{Y}$ de Roux há locais mais propícios ao vazamento do conteúdo gástrico que refletem em complicações, como no local da anastomose gastrojejunal, linha de grampeamento da bolsa gástrica, linha de grampeamento na alça em Roux, jejunojejunostomia, estômago excluso e linha gástrica de grampos remanescentes. Isso tange ao maior conhecimento e atenção do cirurgião para estes locais propícios. Outro ponto de checagem da equipe médica é quanto a integridade do grampeador e dos grampos em cada disparo, pois se um grampo se solta em um dos lados e fica preso ao outro o restante da carga de grampos é danificada, selando em uma das extremidades e abrindo na outra, o que leva à deiscência da linha anastomótica (ACQUAFRESCA PA, et al., 2015).

Após o grampeamento, a utilização de material biológico é eficaz pois diminui a taxa de vazamento e reduz o risco de sangramento nos locais anastomosados. O selante de fibrina tem função selante e adesiva o que diminui vazamentos (ACQUAFRESCA PA, et al., 2015). A integridade das anastomoses deve ser verificada no intra-operatório, através de sonda orogástrica com aplicação de azul de metileno ou insuflação de ar. Outra opção é a utilização de um gastroscópio flexível com anastomose submersa. As suturas ou grampeamentos não devem ser feitos sob tensão pois resultam em estresse tecidual e podem levar à deiscência dos pontos (ACQUAFRESCA PA, et al., 2015).

Além dos cuidados referentes à aplicação da técnica cirúrgica adequada, estudos mostraram que pacientes com classificação pré-operatória ASA3 e ASA4; ou/e submetidos à tempo cirúrgico superior à 2 horas, ou com uma maior taxa de procedimentos concomitantes - colocação de tubo de gastrostomia, ressecção intestinal, esplenectomia e lise de aderências - apresentam maior taxa de complicações (ABRAHAM CR, et al., 2015). Outros fatores associados a complicações pós-operatórias são: idade superior há 50 anos, diabetes mellitus insulino-dependente, uso de hormônios esteróides, sexo feminino, insuficiência cardíaca congestiva, doença pulmonar, artrite reumatóide, histórico de abuso de substâncias e psicose/depressão. Portanto, é necessário atenção a estes fatores no pré-operatório (SHUBECK S, et al., 2018).

\section{CONSIDERAÇÕES FINAIS}

Por meio da análise bibliográfica, evidenciou-se a importância do procedimento cirúrgico Bypass Gástrico em Y de Roux, visto que ele está associado com o tratamento da obesidade com perda de peso significativa, redução da morbidade e mortalidade. Para evitar complicações cirúrgicas tardias observa-se que o manejo 
cirúrgico adequado está relacionado ao melhor prognóstico do paciente. Foram expostas as principais complicações após o BGYR, como também as estratégias para evitá-las. Concomitantemente, o estudo possibilita maior agilidade no diagnóstico de possíveis complicações após o procedimento de BGYR, ao evidenciar os locais mais propícios de ocorrências e suas causas. Por fim, espera-se que a revisão bibliográfica contribua na melhor forma de abordagem a evitar complicações pós Bypass Gástrico em $Y$ de Roux.

\section{REFERÊNCIAS}

1. ABRAHAM CR, et al. Predictors of Hospital Readmission after Bariatric Surgery. J Am Coll Surg. 2015; 221(1): $220-227$.

2. ACQUAFRESCA PA, et al. Complicações cirúrgicas precoces após bypass gástrico: revisão da literatura. ABCD. Arquivos Brasileiros de Cirurgia Digestiva, 2015; 28(1): 74-80.

3. BAILLY L, et al. Anemia and Bariatric Surgery: Results of a National French Surveyon Administrative Data of 306,298 Consecutive Patients Between 2008 and 2016. IFS: Obesity Surgery, 2018; 28: 2313-2320

4. BARROS F, et al. Comparação Da Perda De Peso Após Sleeve E Bypass Gástrico Em Y-de-roux: Revisão Sistemática. Abcd. Arquivos Brasileiros de Cirurgia Digestiva, 2019; 32(4): 1-4

5. BARROS F, et al. Trombose venosa portomesentérica após cirurgia bariátrica: série de casos. Revista do Colégio Brasileiro de Cirurgiões, 2020; 47: 1-7

6. EDERVEEN JC, et al. Diagnóstico de hérnia interna após derivação gástrica laparoscópica em Y de Roux: utilidade da revisão sistemática de tomografias usando dez sinais. Eur Radiol. 2018; 28 (9): 3583-3590.

7. GUIRAT A, ADDOSSARI H. One Anastomosis Gastric Bypass and Risk of Cancer. Obesity Surgery. Springer Science + Business Media LLC, 2018; 28(5): 1441-1444.

8. JARA R. Cierre de brechas mesentéricas em bypass gástrico laparoscópico. Revista Chil Cir. 2017; 69(5): $371-375$

9. KHORGAMI Z, et al. Predictors of readmission after laparoscopic gastric by-pass and sleeve gastrectomy: a comparative analysis of ACS-NSQIP data base. Surg Endosc. 2016; 30(1): 2342-2350.

10. KORNERUP L, et al. Early changes in vitamin B12 up take and biomarker status following Roux-en-Y gastric bypass sand sleeve gastrectomy. Clinical Nutrition, 2019; 38: 906-911.

11. MALA T, HOGESTOL I. Abdominal Pain After Roux-En-Y Gastric Bypass for Morbid Obesity. Scandinavian Journal of Surgery. 2018; 107(4): 277-284.

12. MARAMBIO A. et al. Úlcera marginal Perforada Post BYPASS gástrico laparoscópio. RevChil Cir. 2015; 67(1): 51-56.

13. PYKE O, et al. Marginal ulcer continues tobe a major source of morbidity over time following gastric bypass. Surg Endosc 2019; 33, 3451-3456.

14. MARTI-GELONCH L, et al. Gastrectomía vertical versus by-pass gástrico en $Y$ de Roux: resultados a corto y medioplazo. Revista de cirugía, 2019; 71(1): 29-34

15. NIRUJOGI V, ZOPFI K. Considerações nutricionais para cirurgia plástica em paciente após cirurgia bariátrica. Rev. Bras. Cir. Plást. 2015; 30(2): 295-302.

16. PALERMO M, et al. Late surgical complications after gastric by-pass: a literature review. ABCD. Arquivos Brasileiros de Cirurgia Digestiva, 2015; 28(2): 139-143.

17. SAMPAIO-NETO J, et al. Complicações relacionadas ao bypass gástrico realizado com calibres gastrojejunais diferentes. ABCD. Arquivos Brasileiros de Cirurgia Digestiva, São Paulo 29, 12-14, 2016.

18. Sociedade Brasileira de Cirurgia Bariátrica e Metabólica. Consenso Bariátrico; 2008.

19. SHUBECK S, et al. Long-term Out comes Following Bariatric Surgery. JAMA. 2018;319(3): 302-303.

20. SOUZA N, et al. Impacto nutricional da cirurgia bariátrica: estudo comparativo do Bypass gástrico em $Y$ de Roux e do Sleeve entre pacientes dos sistemas público e privado de saúde. Rev. Col. Bras. Cir, 2020; 47: 01-13.

21. STOLL A, et al. Early Postoperative complications in Roux-en-Y gastric bypass. ABCD. Arquivos Brasileiros de Cirurgia Digestiva, 2016; 29(1): 72-74.

22. TELEM DA, et al. Hospital admissions greater than 30 days following bariatric surgery: patient and procedure matter. Surg Endosc. 2015; 29(6):1310-1315.

23. US DE PAZ G, PARRAGUEZ JE. Complicaciones tardías em cirugía bariátrica. Rev Guatem Cir. 2015; 21: 1-16.

24. VIEIRA E. Complicações decorrentes de cirurgia bariátrica em pacientes atendidos em um hospital no sul de Santa Catarina. ACM arq. Catarin, 2018; 47(3): 74-84.

25. WRZESINSKI A, et al. Complicações que necessitaram de manejo hospitalar no Pós-operatório de cirurgia bariátrica. ABCD, arq. bras. cir. dig.2015; 28(1): 3-6.

26. XIMENES MAS, et al. Achados tomográficos na hérnia de Petersen como complicação de cirurgia bariátrica com bypass gástrico em Y de Roux. Departamento de Imagem do Hospital Albert Einsten - HIAE. einsten. 2008; 6(4):452-8.

27. ZEVE JLM, et al. Técnicas em cirurgia bariátrica: uma revisão da literatura. Revista Ciência \& Saúde. 2012;5(2):132-40.

28. ZORRON R, et al. Evolução do complexo para o simples: procedimentos revisionais e endoscópicos após cirurgia bariátrica. $A B C D$, arq. bras. cir. Dig, 2016; 29(1): 128-133. 